\title{
Discovery of two M 32 twins in Abell 1689^
}

\author{
S. Mieske ${ }^{1,2}$, L. Infante ${ }^{2}$, M. Hilker ${ }^{1}$, G. Hertling ${ }^{2}$, J. P. Blakeslee ${ }^{3}$, N. Benítez ${ }^{4}$, H. Ford ${ }^{3}$, and K. Zekser ${ }^{3}$ \\ 1 Sternwarte der Universität Bonn, Auf dem Hügel 71, 53121 Bonn, Germany \\ e-mail: smieske@astro.uni-bonn.de \\ 2 Departamento de Astronomía y Astrofísica, Pontificia Universidad Católica de Chile, Casilla 306, Santiago 22, Chile \\ 3 Department of Physics and Astronomy, Johns Hopkins University, 3400 North Charles Street, Baltimore, MD 21218, USA \\ ${ }^{4}$ Instituto de Astrofísica de Andalucía (CSIC), Camino Bajo de Huétor 24, Granada 18008, Spain
}

Received 3 November 2004 / Accepted 9 December 2004

\begin{abstract}
The M 31 satellite galaxy M 32 has long been considered an object of unique properties, being the most extreme example of the very rare compact elliptical galaxy class. Here we present the spectroscopic discovery of two M 32 twins in the massive galaxy cluster Abell 1689. As these objects are so rare, this is an important step towards a better understanding of the nature of compact galaxies. The two M 32 twins had first been detected within our photometric search for ultra compact dwarf galaxy (UCDs) candidates in A1689 (Mieske et al. 2004b, AJ, 128, 1529) with the Advanced Camera for Surveys (ACS). Their luminosities $\left(M_{V} \simeq-17 \mathrm{mag}\right)$ are very similar to M 32 and their surface brightness profiles are consistent with that of M 32 projected to A1689's distance. From our ACS imaging we detect several fainter compact galaxy candidates with luminosities intermediate between M 32 and the Fornax UCDs. If spectroscopically confirmed as cluster members, this would almost close the gap in the mag- $\mu$ plane between the region of UCDs and the compact ellipticals, implying a sequence of faint compact galaxies well separated from that of dwarf ellipticals.
\end{abstract}

Key words. galaxies: clusters: individual: Abell 1689 - galaxies: dwarf - galaxies: structure - galaxies: interactions

\section{Introduction}

\subsection{Compact ellipticals (cEs) and ultra compact dwarfs (UCDs)}

In the magnitude-surface brightness plane of stellar systems, early-type galaxies populate a well defined sequence of increasing central surface brightness with increasing total luminosity (Jerjen \& Binggeli 1997; Infante et al. 2003; Hilker et al. 2003; Karick et al. 2003; Graham \& Guzman 2003), extending over about ten magnitudes. This sequence is folded up only at the very bright end due to core formation in the most luminous giant ellipticals (Faber et al. 1997). Naturally, galaxies that fall well outside this sequence are very attractive objects to study. Probably the most extreme outlier towards high surface brightness at low luminosity is the compact elliptical (cE) galaxy M 32 (e.g. Faber 1973; Kormendy 1985, 1987; Nieto \& Prugniel 1987). It has $M_{V} \simeq-17 \mathrm{mag}$ (Graham 2002) and an effective surface brightness $\mu_{\text {eff }}$ about four mag brighter than dwarf ellipticals (dEs) of comparable $M_{V}$. The effective radius of its bulge is only $\sim 100 \mathrm{pc}$. Systematic searches for M 32 analogs in other nearby clusters have up to now not proven successful (e.g. Ziegler \& Bender 1998;

^ Based on observations obtained at the European Southern Observatory, Chile (Observing Programme 273.B-5008).
Drinkwater \& Gregg 1998). There are only few faint elliptical galaxies with roughly similar properties to M 32 (e.g. NGC 4486B and CCC 70, see Jerjen \& Dressler 1997), but M 32 still remains the faintest and most compact one.

As most of these cEs are found close to more luminous giant galaxies, discussions about their origins have focussed on tidal effects from their neighbours. As progenitor galaxies for M 32, bulges of late type spirals (Bekki et al. 2001) have been suggested as well as genuine low luminosity ellipticals (Faber 1973). Choi et al. (2002) find that a normal elliptical galaxy cannot have been a precursor of M 32, but that its precursor must have been intrinsically compact. Graham (2002) finds that M 32's surface brightness profile can be best fit by a compact Sersic bulge plus a low surface brightness exponential disk. These two findings support the idea that M 32 is the compact bulge of a stripped spiral rather than a normal elliptical.

Going about a factor of 100 in luminosity and 10 in effective radius lower, one enters the regime of the so-called "ultra compact dwarf galaxies" (UCDs), discovered in the Fornax cluster by Hilker et al. (1999) and Drinkwater et al. (2000). These overluminous star clusters have $-11>M_{V}>-13 \mathrm{mag}$ (Mieske et al. 2004a) and are more concentrated towards the cluster centre than the average dwarf galaxy population. Also for UCDs, tidal stripping of nucleated dwarf galaxies has been proposed as a possible origin (Bekki et al. 2003). 
Table 1. Properties of the spectroscopically investigated UCD candidates in Abell 1689. "CG" in the comment column stands for "compact galaxy". The spectrum of candidate 4 is partially contaminated by a neighbouring irregular galaxy with unkown redshift, see Fig. 1, showing several weak cross-correlation peaks within $0<z<0.30$. The spectrum of candidate is partially contaminated by a dwarf galaxy member of Abell 1689, see Fig. 1, showing several weak cross-correlation peaks within $0.15<z<0.20$.

\begin{tabular}{|c|c|c|c|c|c|c|}
\hline Candidate & RA[2000] & $\bar{D} \operatorname{Dec}[2000]$ & $\bar{i}$ & $(g-i)$ & $z_{\text {spec }}$ & Comment \\
\hline 1 & $13: 11: 32.75$ & $-01: 19: 48.9$ & 22.18 & 1.64 & $0.1859 \pm 0.0008$ & new name: $\mathrm{CG}_{\mathrm{A} 1689,1}$ \\
\hline 2 & $13: 11: 29.90$ & $-01: 19: 22.5$ & 22.38 & 1.75 & $75 \pm 260 \mathrm{~km} \mathrm{~s}^{-1}$ & Star \\
\hline 3 & $13: 11: 33.11$ & $-01: 20: 05.5$ & 22.77 & 1.74 & $0.2014 \pm 0.0007$ & new name: $\mathrm{CG}_{\mathrm{A} 1689,2}$ \\
\hline 4 & 13:11:31.09 & $-01: 21: 14.8$ & 23.24 & 1.51 & $0.00<z_{\text {spec }}<0.30$ & Possible member (weak, blended) \\
\hline 5 & $13: 11: 25.73$ & $-01: 21: 42.1$ & 23.80 & 1.67 & $0.15<z_{\text {spec }}<0.20$ & Possible member (weak, blended) \\
\hline
\end{tabular}

\subsection{UCD candidates in Abell 1689}

In the densest environments, tidal stripping should occur more frequently than anywhere else. Therefore we undertook a photometric search for UCD candidates in the very massive galaxy cluster Abell 1689 ( $z=0.183, m-M \simeq 39.75 \mathrm{mag}$ ), see Mieske et al. (2004b). This search was based on deep high resolution ACS images, see Fig. 1. UCD candidates were selected based on their compact morphology, brightness and $(g-i)$ colour. In addition, their photometric redshift was demanded to be smaller than 0.5, as derived using the BPZ code by Benítez (2000). The search resulted in the discovery of several very luminous UCD candidates in the luminosity range between Fornax UCDs and M 32. Two of the brightest UCD candidates ( $i \simeq 22.5 \mathrm{mag}, M_{V} \simeq-17 \mathrm{mag}$ ) are marginally resolved, implying $r_{\text {eff }} \simeq 300$ pc at Abell 1689's distance, similar to M 32 .

In this letter, we present the spectroscopic follow-up of the five brightest UCD candidates in Abell 1689.

\section{Spectroscopic results}

The spectroscopic observations have been obtained with Director's Discretionary Time (DDT) program 273.B-5008. The spectra were taken with the VLT/FORS2 mask exchange unit MXU, targeting the five brightest UCD candidates in Abell 1689 from Mieske et al. (2004b) and several other objects of interest (stellar super cluster candidates, dwarf galaxies, etc.). The slit-width was $1^{\prime \prime}$, the resolution was about $6 \AA$ /pixel at a spatial scale of 0.2 /pixel. The PSF-FWHM was about $0.8^{\prime \prime}$. The total on-source integration time was $6500 \mathrm{~s}$. The resulting $S / N$ per pixel in the best exposed wavelength range around $6000 \AA$ was about 15 for the brightest UCD candidate $(i=$ $22.2 \mathrm{mag}$ ) and about 6 for the faintest one ( $i=23.8 \mathrm{mag})$. The UCD candidate spectra are shown in Fig. 3, the derived redshifts in Table 1.

We confirm the cluster membership for UCD candidates Nos. 1 and 3. The redshift of candidate No. 2 is consistent with a galactic foreground star. The spectra of the two faintest UCD candidates Nos. 4 and 5 were partially blended with neighbouring galaxies, inhibiting an unambiguous redshift determination. Both spectra exhibit several weak cross correlation peaks in a range of redshifts, including peaks corresponding to A1689's distance. Longer spectroscopic integrations and better seeing are needed to definitely confirm or rule out their cluster membership. Judging from the thumbnail
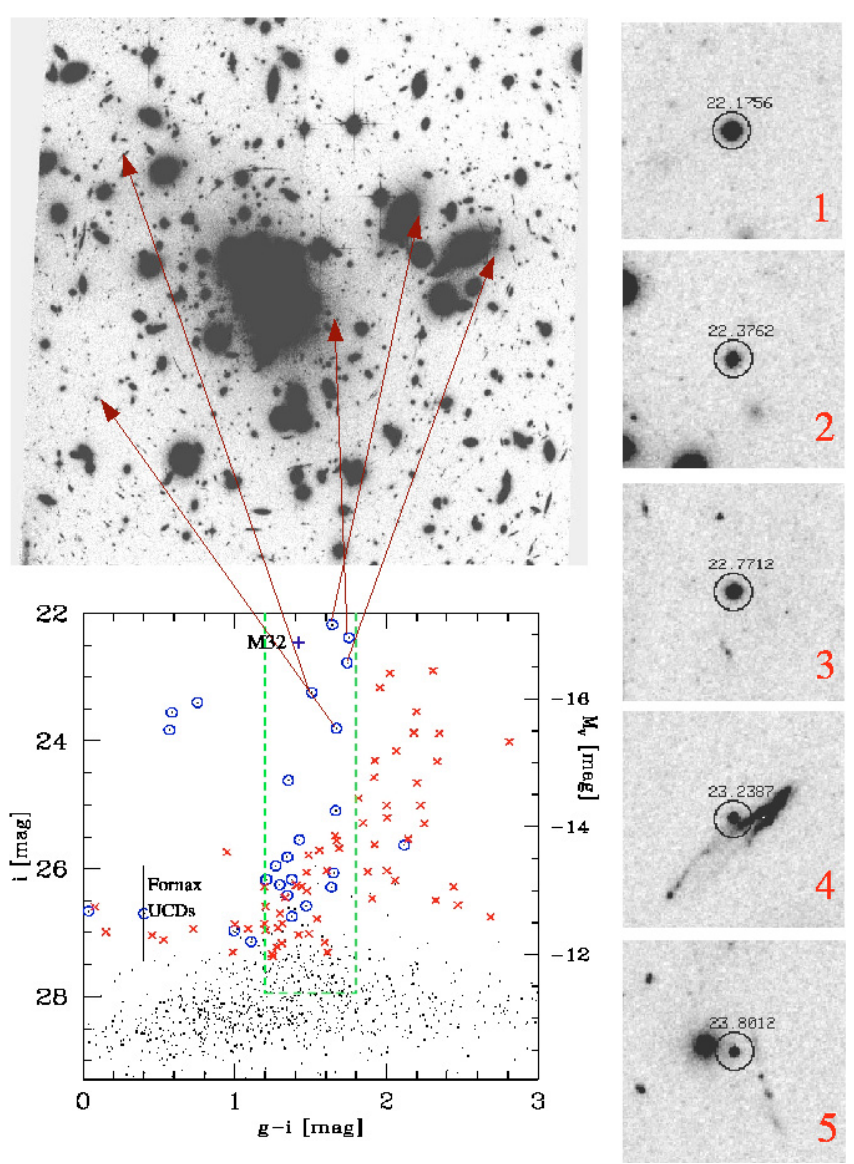

Fig. 1. Top: ACS image of Abell 1689 plus thumbnails of the five brightest UCD candidates, numbered by decreasing luminosity according to Table 1 . The thumbnail width is $7^{\prime \prime}$. Bottom: CMD of UCD candidates in Abell 1689. Circles: Photometric redshift $z_{\text {phot }}<0.5$. Crosses: $z_{\text {phot }}>0.5$. The dashed vertical lines indicate the colour range of Fornax UCDs, the vertical tick their apparent luminosities if shifted to A1689's distance (Mieske et al. 2004a,b). The approximate location in the CMD of M 32 at A1689's distance is indicated by the cross (Mateo 1998).

image in Fig. 1, candidate No. 4 might also be part of a lensed background galaxy.

A note on nomenclature: the absolute luminosities of the two confirmed A1689 UCD candidates are almost identical to M 32, such that one might also call them "compact elliptical" (cE) or "compact dwarf elliptical" (cdE). We choose to assign the two cluster members the more general term "compact galaxies" (CGs) or "M 32-like galaxies". 

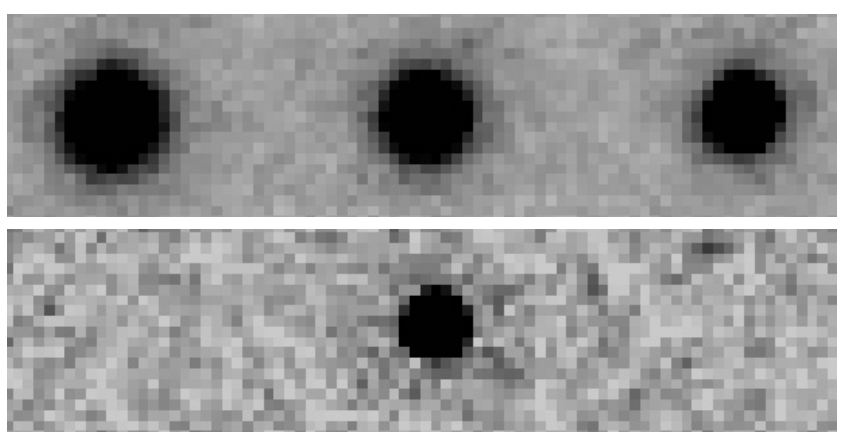

Fig. 2. Top panel: morphological comparison between (from left to right): $\mathrm{CG}_{\mathrm{A} 1689,1}(i=22.2 \mathrm{mag}) ; \mathrm{M} 32$ modelled according to the fit by Graham (2002), projected to A1689's distance and seeing convolved $(i=22.4 \mathrm{mag}) ; \mathrm{CG}_{\mathrm{A} 1689,2}(i=22.8 \mathrm{mag})$. Bottom panel: an unresolved point source ( $i=22.4 \mathrm{mag})$. The image excerpts have the same intensity cuts and are taken from deep ACS $i$-band exposures of Abell 1689. There is a higher noise level for the lower image because the point source originally is about $1.5 \mathrm{mag}$ fainter and was scaled up to fall in the luminosity range of the M 32-like objects.

\section{Comparison between M 32 and compact galaxies in Abell 1689}

In Figs. 2 and 4 the morphological appearance and surface brightness (SB) profiles of the two A1689 compact galaxies (CGs) is compared with a seeing convolved, simulated SB model of M 32, as taken from the fit by Graham (2002). This fit consists of a central bulge component described by a Sersic profile with $n=1.5$ and $r_{\text {eff }}=105 \mathrm{pc}$ and an underlying exponential disk with exponential scale length $h=480 \mathrm{pc}$ $\left(r_{\mathrm{eff}}=806 \mathrm{pc}\right)$. Ellipticity was adopted as zero. The PSF profile used for convolution was adopted as a Moffat function with $F H W M=0.095^{\prime \prime}$ and $\beta=2.5$. For the simulation, an an-

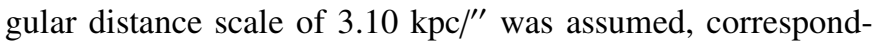
ing to a mean cluster redshift of $z=0.1832$ (NED database), $H_{0}=70 \mathrm{~km} \mathrm{~s}^{-1} \mathrm{Mpc}^{-1}, \Omega_{\mathrm{M}}=0.3$ and $\Omega_{\Lambda}=0.7$.

On the ACS image in Fig. 2, the two A1689 CGs and the simulated M 32 appear very similar and are notably more extended than a pure stellar source of comparable luminosity. In the surface brightness plots of Fig. 4, this is confirmed. When fitting an exponential profile to the outer parts of the two CG profiles that are mostly unaffected by the seeing, effective radii in the range $700-800 \mathrm{pc}$ are determined, in agreement with the scale size of M 32's exponential disk. In the lower panel of Fig. 4, it is shown that also a single Sersic profile with $r_{\text {eff }}=300 \mathrm{pc}$ and $n=1.85$ fits quite well the observed $\mathrm{CG}$ profiles. In order to explore the acceptable range for $r_{\mathrm{eff}}$ in the case of a single component, we used the ISHAPE task (Larsen 1999). As input PSFs we used three different unresolved sources in the $i$ exposure of the ACS image, with PSF-FWHM between 0.09 and $0.10^{\prime \prime}$. For the intrinsic profile shape we adopted King profiles with concentration parameter between 5 and 30 and a Moffat profile with $\beta=2.5$. The mean and scatter of the fitted $r_{\text {eff }}$ obtained with different models and PSFs is $370 \mathrm{pc} \pm 180 \mathrm{pc}$ for $\mathrm{CG}_{\mathrm{A} 1689,1}$ and $225 \mathrm{pc} \pm$ $75 \mathrm{pc}$ for $\mathrm{CG}_{\mathrm{A} 1689,2}$. All of the profile fits have a comparable $\chi^{2}$. Doing the same procedure for the simulated, seeing convolved

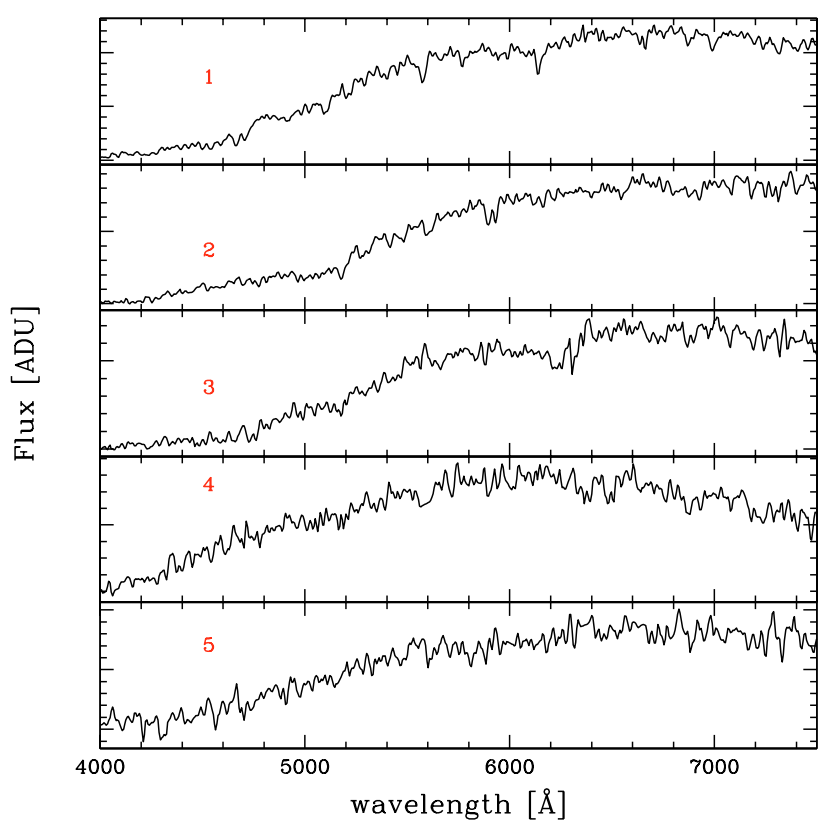

Fig. 3. Wavelength calibrated spectra of the five UCD candidates, ordered from top to bottom by decreasing $i$ luminosity. The spectra are not flux-calibrated. Candidates 1 and 3 have spectroscopic redshifts consistent with A1689. The H\&K break is at about $4700 \AA$ for candidate 1 and 4800 Å for candidate 3 .

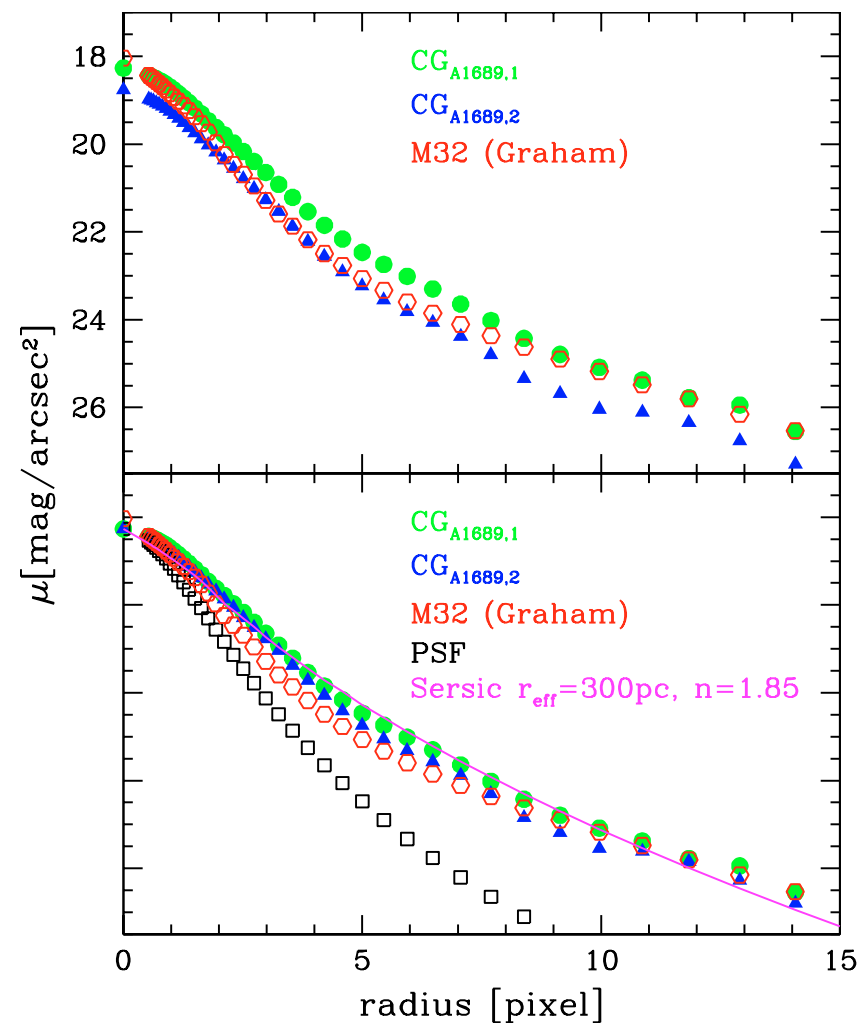

Fig. 4. Comparison of surface brightness (SB) profiles, with scale $0.05^{\prime \prime} /$ pixel, or $155 \mathrm{pc} / \mathrm{pixel}$ at Abell 1689's distance. Upper panel: $\mathrm{CG}_{\mathrm{A} 1689,1}$ (filled circles), $\mathrm{CG}_{\mathrm{A} 1689,2}$ (filled triangles), M 32's profile from the fit by Graham (2002), projected to A1689's distance and PSF convolved (open hexagons). Lower panel: the three SB profiles from the upper panel normalised to the same peak intensity plus the normalised PSF profile (Moffat profile with 0.095" FWHM). The solid line is a PSF convolved Sersic profile with $r_{\mathrm{eff}}=300 \mathrm{pc}$ and $n=1.85$. 


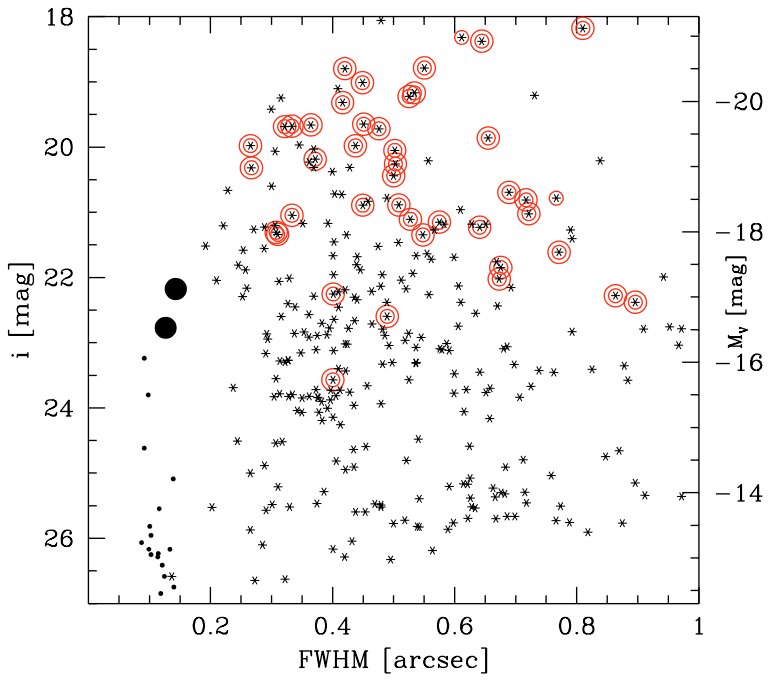

Fig. 5. Size-luminosity distribution of normal dwarf galaxies and UCD candidates in Abell 1689. SExtractor FWHM is plotted vs. $i$-band luminosity for sources with $z_{\text {phot }}<0.5$ in the indicated magnitude range. The PSF-FWHM is about $0.10^{\prime \prime}$. The dots are the unresolved UCD candidates, defined by a star-classifier larger than 0.6 (see Mieske et al. 2004b). The asterisks indicate the resolved dwarf galaxy candidates, defined as having SExtractor star-classifier smaller than 0.6. The larger filled circles indicate the two confirmed M 32 twins. The open circles indicate galaxies with a measured spectroscopic redshift (Czoske 2004), double circles are those with $0.16<$ $z<0.22$ (redshift range of Abell 1689).

M 32 image yields an intrinsic effective radius of $225 \mathrm{pc} \pm$ $75 \mathrm{pc}$.

\section{Discussion and conclusions}

Objects like M 32 are extremely rare. In this letter we have presented the discovery of two M 32 analogs in Abell 1689, an extremely massive cluster which is also known as the strongest lens on the sky $\left(M \simeq 1 \times 10^{15} M_{\odot}\right.$, see King et al. 2002). This is consistent with the hypothesis that deep potential wells favour the creation of such compact galaxies. $\mathrm{CG}_{\mathrm{A} 1689,1}$ has a relative velocity of $-4800 \mathrm{~km} \mathrm{~s}^{-1}$ with respect to the closest projected cluster giant elliptical and $+1500 \mathrm{~km} \mathrm{~s}^{-1}$ to the second nearest one, values which are consistent with the high velocity dispersion of Abell 1689 (Czoske 2004). $\mathrm{CG}_{\mathrm{A} 1689,2}$ has a much smaller velocity difference of about $100 \mathrm{~km} \mathrm{~s}^{-1}$ to its closest neighbour, comparable to the relative velocity between $M 31$ and M 32. This fits into a picture where both galaxy-satellite interactions and tidal interaction with the overall cluster potential can lead to the creation of compact galaxies. If this is true then there should exist a continuous sequence of compact galaxies between the fainter Fornax-UCD types and brighter M 32-types, especially in an extremely massive cluster like Abell 1689. As is shown in Figs. 1 and 5 and outlined in detail in Mieske et al. (2004b), there indeed is a population of compact galaxy candidates with luminosities intermediate between the Fornax UCDs and M 32, in addition to the two brighter confirmed M 32 analogs. The definite confirmation of their membership with spectroscopy is needed to exclude them being foreground stars or background galaxies. If confirmed as cluster members, this would fill a previously unpopulated region in the mag- $\mu$ plane of stellar systems: a sequence of faint compact galaxies parallel to the dwarf galaxy sequence but offset towards higher surface brightness/smaller scale length. Figure 5 illustrates this: there is a notable gap in FWHM between the unresolved UCD candidates and the resolved "normal" dwarf galaxy candidates. Our sample is complete across this gap, i.e. the gap is not caused by selection effects.

The next step will be to perform a spectroscopic census of the entire compact galaxy population in Abell 1689, extending from the brighter $\mathrm{cE}$ candidate galaxies not observed by Czoske (2004) to several mag fainter than the two newly discovered M 32 twins. Furthermore, the results presented here encourage us to propose a search for compact galaxy candidates in other massive clusters.

Acknowledgements. We thank the referee M. Drinkwater for his comments which helped to improve the paper. We thank the ESO User Support Group for carrying out the DDT spectroscopy (program 273.B-5008) in service mode. ACS was developed under NASA contract NAS 5-32864. SM was supported by DAAD Ph.D. grant Kennziffer D/01/35298 and DFG Projekt Nr. HI 855/1-1. LI would like to acknowledge support from "proyecto Fondap \# 15010003".

\section{References}

Bekki, K., Couch, W. J., Drinkwater, M. J., \& Gregg, M. D. 2001, ApJ, 557, L39

Bekki, K., Couch, W. J., Drinkwater, M. J., \& Shioya, Y. 2003, MNRAS, 344, 399

Benítez, N. 2000, ApJ, 536, 571

Choi, P. I., Guhathakurta, P., \& Johnston, K. V. 2002, AJ, 124, 310

Czoske, O. 2004, to appear in Proc. of IAU Coll. 195, ed. A. Diaferio et al., in preparation [arXiv: astro-ph/0403650]

Drinkwater, M. J., \& Gregg, M. D. 1998, MNRAS, 296, L15

Drinkwater, M. J., Jones, J. B., Gregg, M. D., \& Phillipps, S. 2000 , PASA, 17, 227

Faber, S. M. 1973, ApJ, 179, 423

Faber, S. M., Tremaine, S., Ajhar, E. A., et al. 1997, AJ, 114, 1771

Graham, A. W. 2002, ApJ, 568, L13

Graham, A. W., \& Guzman, R. 2003, AJ, 125, 2936

Hilker, M., Infante, L., Vieira, G., Kissler-Patig, M., \& Richtler, T. 1999, A\&As, 134, 75

Hilker, M., Mieske, S., \& Infante, L. 2003, A\&A, 397, L9

Infante, L., Mieske, S., \& Hilker, M. 2003, Ap\&SS, 285, 87

Jerjen, H., \& Binggeli, B. 1997, ASP Conf. Ser. 116, ed. M. Arnaboldi, G. S. Da Costa, \& P. Saha, 239

Jerjen, H., \& Dressler, A. 1997, A\&AS, 124, 1

Karick, A. M., Drinkwater, M. J., \& Gregg, M. D. 2003, MNRAS, 344,188

King, L. J., Clowe, D. I., \& Schneider, P. 2002, A\&A, 383, 118

Kormendy, J. 1985, ApJ, 295, 73

Kormendy, J. 1987, Proc. IAU Symp. 127 (Dordrecht: D. Reidel Publishing Co.), 17

Larsen, S. S. 1999, A\&AS, 139, 393

Mateo, M. 1998, ARA\&A, 36, 435

Mieske, S., Hilker, M., \& Infante, L. 2004a, A\&A, 418, 445

Mieske, S., Infante, L., Benítez, N., et al. (ACS GTO team) 2004b, AJ, 128,1529

Nieto, J.-L., \& Prugniel, P. 1987, A\&A, 186, 30

Ziegler, B. L., \& Bender, R. 1998, A\&A, 330, 819 